Revue d'histoire de l'Amérique française

ZAS REVUE D.HISTOIRE DE L'AMÉRIQUE FRANÇAISE

\title{
Marie de l'Incarnation et le climat spirituel de la Nouvelle-France
}

\section{André Rayez}

Volume 16, numéro 1, juin 1962

URI : https://id.erudit.org/iderudit/302167ar

DOI : https://doi.org/10.7202/302167ar

Aller au sommaire du numéro

Éditeur(s)

Institut d'histoire de l'Amérique française

ISSN

0035-2357 (imprimé)

1492-1383 (numérique)

Découvrir la revue

Citer cet article

Rayez, A. (1962). Marie de l'Incarnation et le climat spirituel de la Nouvelle-France. Revue d'histoire de l'Amérique française, 16(1), 3-36. https://doi.org/10.7202/302167ar d'utilisation que vous pouvez consulter en ligne.

https://apropos.erudit.org/fr/usagers/politique-dutilisation/ 


\section{MARIE DE L'INCARNATION ET LE CLIMAT SPIRITUEL DE LA NOUVELLE-FRANCE}

La période qui s'écoule entre l'arrivée au Canada de Marie de l'Incarnation, 1639 , et sa mort, 1672, a paru, à plus d'un historien, ${ }^{1}$ revêtir un caractère particulier, exceptionnel, voire héroïque. Naguère, Monsieur Guy Frégault écrivait: «De la baie d'Hudson au golfe du Mexique et de Terre-Neuve à l'ouest des grands lacs, partout la présence canadienne s'est manifestée par le travail de fortes équipes de combattants, d'explorateurs, de commerçants, de missionnaires et d'organisateurs. Tout cela a été l'œuvre d'un grand siècle, et c'est le XVII'. ${ }^{2}$

J'ai l'honneur et la joie de vous parler de cette poignée d'hommes et de femmes qui choisirent de vivre et de mourir au Canada, pour de tout autres mobiles que le combat, l'exploration ou le commerce. L'histoire de leur vie spirituelle et l'histoire de la vie des chrétientés qu'ils ont tenté de fonder et de faire vivre, seules, nous retiendront.

La sociologie religieuse donnerait sans doute une explication suffisante à certains faits des origines du Canada. Toutefois, lorsqu'elle tend à les réduire à des phénomènes économiques ou politiques, sociaux ou folkloriques, elle reste, en bien des cas, partielle, superficielle même, erronée parfois. L'explication des mobiles profonds de l'action ne serait-elle pas, en fin de compte, le dernier mot de l'histoire ? Aussi, ai-je l'ambition, beaucoup

1 Fr.-X. Garneau, dans son Histoire du Canada depuis sa découverte jusqu'à nos jours, si tendancieux soit-il lorsqu'il juge en «philosophe » les missionnaires, reconnaît leur bienfaisante et durable influence (6 ${ }^{\mathrm{e}}$ éd., Paris, 1914), III: ch. 1. Francis Parkman, américain passé de l'unitarisme à l'agnosticisme, signale, lui aussi, cette influence: «Plus encore que la puissance royale, l'Eglise de Rome a modelé le caractère et préparé les destinées de cette colonie. Elle a été sa nourrice et, pour tout dire, sa mère» (The Old Régime in Canada (Boston, 1874), 400). Voir aussi, de Parkman, The Jesuits in North America in the seventeenth century (Boston, 1867).

2 Cahiers de l'Académie canadienne-française, II: Histoire, (Montréal, 1957), 55. 
trop audacieuse sans doute dans l'état actuel des recherches, ${ }^{3}$ d'esquisser devant vous une page de l'histoire de la spiritualité du Canada au temps de Marie de l'Incarnation. En me tenant aussi près que possible des textes mêmes de l'ursuline, peut-être mériterais-je davantage votre indulgence ? 4

Pour bien comprendre, en effet, la vie, l'œuvre et la spiritualité de Marie de l'Incarnation, il convient de les situer dans

3 Il semble que le sujet n'ait pas trouvé son historien. La spiritualité au Canada a été effleurée en passant et en des secteurs fort limités par les biographes des principaux personnages qui ont illustré les origines du pays. Un tableau objectif d'ensemble manque encore. Sans doute, après Georges Goyau, dont Les origines religieuses du Canada (Paris, 1924, 1934, 1939) demeurent une très belle esquisse, le mieux préparé parmi les derniers historiens eût été dom Albert Jamet. Recherches d'archives, éditions et confrontations des textes, histoire religieuse générale, rien ne l'arrêtait. Il $\mathrm{y}$ joignait l'indispensable sympathie pour son sujet, sans quoi tout travail comme toute conclusion deviennent inhumains.

4 Marie de l'Incarnation a composé divers écrits spirituels. En 1633, elle adressait au P. Georges de La Haye, son directeur, une Relation de sa vie intérieure, dont l'original est perdu; des fragments importants ont été publiés par son fils Claude Martin. Une seconde Relation fut rédigée en 1654, à la demande de Jérôme Lalemant, son directeur au Canada, et envoyée à son fils; une copie en est conservée au monastère des Ursulines de Trois-Rivières; dom Claude Martin a reproduit intégralement cette Relation, sans doute d'après l'original, disparu également. Ce qui reste de la première Relation et la seconde (d'après la copie de Trois-Rivières) ont été édités critiquement par dom Albert Jamet dans les tomes 1 et 2 des Ecrits spirituels et historiques de Marie de l'Incarnation, accompagnés d'une abondante et très précieuse annotation; ces deux textes ont été aussi publiés par dom Jamet, sous le titre, Le témoignage de Marie de l'Incarnation (Paris, Beauchesne, 1932), mais avec une annotation fort restreinte.

La correspondance de Marie de l'Incarnation a été très abondante; une bonne partie est perdue. On ne peut que déplorer la disparition de la correspondance échangée avec Jean de Bernières (1602-1659), son fidèle factotum financier, avec lequel elle se trouvait en étroite communion spiritueile, celle échangée pendant de longues années avec le P. JeanBaptiste de Saint-Jure (1588-1657), qui fut le conseiller attentif des missionnaires, hommes et femmes, du Canada, celle échangée avec plusieurs missionnaires, comme saint Charles Garnier, etc. Les lettres connues aujourd'hui forment cependant un ensemble imposant: lettres à ses directeurs spirituels (le feuillant Raymond de Saint-Bernard notamment), aux Ursulines de Tours, de Dijon, de Paris, de Mons, etc., à des missionnaires (Pail Le Jeune, Antoine-Joseph Poncet, etc.). Sa correspondance avec son fils, de 1639 à 1672 , est le lot le plus remarquable. On peut ajouter à ces écrits spirituels des «Exclamations et élévations 》 (titre donné par dom Jamet), des Méditations et ses Retraites de 1634 et de 1636, une Exposition succincte du Cantique des cantiques.

Claude Martin publia La Vie de la Vénérable Mère Marie de l'Incarnation, première supérieure des Ursulines de la Nouvelle-France, tirée de ses lettres et de ses écrits (Paris, in $-4^{\circ}$ ), 757 pages; des Lettres de la 
le courant religieux de la Nouvelle-France. Nous retrouvons sans peine, chez la fondatrice du Vieux Monastère de Québec, les orientations fondamentales de la spiritualité des meilleurs chrétiens du pays, fidèles comme missionnaires. Marie de l'Incarnation a vécu intensément dans ce climat spirituel; elle a contribué à accentuer certains de ses aspects essentiels. Elle épousa les préoccupations religieuses et spirituelles de son temps, elle y répondit à sa manière, toujours tendue vers un don de plus en plus total à l'Église et à Dieu.

Il semblerait qu'on puisse résumer sommairement les caractéristiques du mouvement spirituel à l'époque de Marie de l'Incarnation en ces trois données :

1) le sens missionnaire et ecclésial, qui en est l'âme, se manifeste

Vénérable Mère Marie de l'Incarnation... (Paris, 1681, in-4 ${ }^{\circ}$ ), 675 pages; des Retraites de la Vénérable ..., avec une Exposition succincte... (Paris, 1682 , in $\left.-12^{\circ}\right), 248$ pages. François-Xavier de Charlevoix a cité abondamment ces textes dans sa Vie de la Mère Marie de l'Incarnation (Paris, 1724); il utilise le manuscrit de Trois-Rivières.

L'abbé Richaudeau a donné une édition des Lettres..., augmentées de huit lettres inédites (2 vol., Paris-Tournai, in- $\left.8^{\circ}, 1876\right)$. Paul Renaudin a publié des extraits, en particulier la seconde Relation, et diverses lettres, dans la collection Maîtres de la spiritualité chrétienne (Paris, Aubier, 1942), précédés d'une introduction: (5-48). Enfin, dom Albert Jamet, bénédictin de Solesmes, a préparé avec grand soin une édition critique des Ecrits spirituels et historiques de Marie de l'Incarnation. Les 4 volumes publiés (in $-8^{\circ}$, Paris - Québec, 1929, 1930, 1935, 1939) comprennent les deux Relations, les Retraites, les Exclamations, l'Exposition succincte, et une partie de la correspondance. L'édition de la correspondance sera achevée par une bénédictine de Saint-Eustache d'après les manuscrits laissés par dom Jamet.

Claude Martin, né à Tours le 2 avril 1619, entra dans la Congrégation de Saint-Maur, à Vendôme, le 15 janvier 1641. Il sera en charge dans différentes maisons à partir de 1652: prieur à Meulan, aux Blanes Manteaux (Paris), à Meulan, à Compiègne, à Angers, à Rouen, à Saint-Denis, à Marmoutier; deux fois assistant du supérieur général; choisi, de fait, comme supérieur général en 1687, il se heurte au veto royal. Il meurt le 9 août 1696. En plus des ouvrages concernant sa mère, Claude Martin publie des Méditations chrétiennes (2 vol., Paris, 1669), une Conduite pour la retraite du mois (Paris, 1670), une Pratique de la Règle de saint Benô̂t (Paris, 1674). Il composa un gros ouvrage de Questions ascétiques, qui furent publiées récemment sous le titre de Conférences ascétiques ( 2 vol., Paris, 1957), des réflexions de retraite à l'usage des supérieurs (Le pasteur solitaire) que l'éditeur a transformé en Perfection du chef (Paris, 1953). Claude Martin, à la fin de sa vie, préparait une réplique à la doctrine de Pierre Nicole sur l'oraison, voir A. Rayez, Le «Traitté de la contemplation》 de dom Claude Martin, dans la Revue d'ascétique et de mystique, 29 (1953): 206-249. Consulter L. Cognet, Dom Claude Martin et le mysticisme français, dans la Revue d'histoire de l'Eglise de France, 43 (1957): 123-149. 
en une spiritualité de service des autres et de Dieu, que nourrissent le désir de la Croix et la hantise du martyre;

2) l'apostolat $d u$ laïcat et spécialement la vocation missionnaire féminine se développent en France et s'épanouissent au Canada dans un dévouement désintéressé au service de la mission;

3) une vie chrétienne qui, malgré ses lacunes, ses déviations et ses misères, est, à des degrés divers, fervente dans la communauté des fidèles et chez les missionnaires; des dévotions très chères entretiennent cette ferveur et des charismes exceptionnels l'apparentent à l'esprit de l'Église naissante.

\section{SENS MISSIONNAIRE}

La vie spirituelle des missionnaires du Canada au $17^{\mathrm{e}}$ siècle, en ses développements les plus profonds, s'explique par le sens étonnamment missionnaire qui anime et informe ces hommes et ces femmes transplantés volontairement au-delà des océans.

La découverte des terres, au $16^{\text {e }}$ siècle, s'était faite au nom de la foi chrétienne, cependant, l'appât de trésors fabuleux attirait trafiquants, découvreurs et soldats. Des missionnaires souvent les accompagnaient, avec des vues autrement désintéressées. Il en sera à peu près de même en Nouvelle-France. Sans refaire ici l'histoire des origines de la colonisation et de ses mobiles, remettons-nous en mémoire quelques-unes des raisons qui ont guidé les expéditions françaises.

Qui ne se souvient de l'humoristique et piquant «Adieu à la France » que Marc Lescarbot fait imprimer à La Rochelle le 3 avril 1606 avant de s'embarquer ?

Prélats que Christ a mis pasteurs de son Eglise,

A qui partant, il a sa parole commise,

Afin de l'annoncer par tout cet univers....,

Quoy done, souffrirez-vous l'ordre du mariage

Sur votre ordre sacré avoir cet advantage

D'avoir eu devant vous le désir, le vouloir,

Le travail et le soin de ce chrétien devoir ? 5

${ }^{5}$ Marc Lescarbot, Histoire de la Nouvelle France $\left(2^{\mathrm{e}}\right.$ éd., Paris, 1611), 513. On ne peut prendre cet Adieu avec plus de sérieux que l'auteur: c'est un passe-temps de voyage ! «Devant qu'arriver à la Rochelle, me tenant quelquefois à quartier de la compagnie, il me print envie de mettre sur mes tablettes un Adieu à la France... \ Il ajoute à la fin de son poème: Pour m'égayer l'esprit ces vers je composois, $\mathrm{Au}$ premier que je vi les murs des Rochelois. 
Les prélats s'étaient préoccupés des «sauvages》 sans attendre la verve moqueuse du navigateur et ils se réjouissaient de voir «l'ordre du mariage» prendre au sérieux le «chrétien devoir » de l'évangélisation. Peut-être auraient-ils souhaité de sa part moins de calculs !

Champlain, lui, remplissait avec sérieux ce « chrétien devoir », et il en rappelait sans hésiter l'esprit et l'obligation à son souverain. En ses Voyages, il s'adresse à Louis XIII pour le louer d'avoir fait ce qu'il lui souhaite le voir faire! C'est « une grâce spéciale de Dieu d'avoir voulu réserver sous vostre règne l'ouverture de la prédication de son Évangille et la cognoissance de son Saint Nom à tant de nations qui n'en avoient jamais oüy parler. ${ }^{6}$ L'explorateur dresse en 1632 un programme pour la réinstallation de la France au Canada. Le souci de l'évangélisation y est premier. «Dans la nouvelle France, déclare-t-il, y a nombre infiny de peuples sauvages... qui... n'ont aucune cognoissance de Dieu. Mais il y a espérance que les religieux qu'on y a menez et qui commencent à s'y establir, y faisant des séminaires, pourront en peu d'années y faire de beaux progrez pour la conversion de ces peuples. C'est le principal soin de sa Majesté...»7

On sait quel mouvement missionnaire fut suscité en France pendant la première moitié $d u 17^{\mathrm{e}}$ siècle par les récits des voyageurs et des missionnaires. Les Relations des Jésuites du Canada connurent un retentissement tellement extraordinaire que dom

6 Voyages et descouvertures (sic) faites en la Nouvelle France... par le Sieur de Champlain (Paris, 1619), reproduits dans les Oeuvres de Champlain ...., éd. C.-H. Laverdière (Québec, 1870), IV: p. V. Plus haut, Champlain écrivait plus nettement encore: «Vous y verrés pareillement quelle et combien grande est l'espérance que nous avons de tant de longs et pénibles travaux que depuis quinze ans nous soustenons pour planter en ce païs l'estendart de la Croix, et leur enseigner la cognoissance de Dieu et gloire de son Sainct Nom, estant nostre désir d'augmenter la charité envers ses misérables créatures.... (p. III).

7 Les voyages de la Nouvelle France occidentale, dicte Canada, faits par le $S^{r}$ de Champlain Xainctongeois..., (Paris, 1632), livre 1, ch. 1; (éd. Laverdière), $\mathrm{V}: 4$. 
Albert Jamet n'hésite pas à y voir «l'un des plus puissants élans missionnaires qui aient jamais traversé la France $\$^{8}$

Ces Relations soulèvent, en effet, dans tout le pays un vaste mouvement de solidarité et de zèle chez les laïcs, chez les religieux et dans les monastères féminins cloîtrés. C'est dans l'enthousiasme qu'on répond aux appels réitérés du Père Paul Le Jeune. La Relation de 1635 signale avec joie: «Il y en a tant qui nous écrivent, et de tant de monastères, et de divers Ordres très réformez, en l'Église, que vous diriez que c'est à qui se mocquera la première des difficultez de la mer, des mutineries de l'Océan et de la barbarie de ces contrées. ${ }^{9}$

En 1637, Le Jeune énumère ces monastères qui se sont ainsi offerts, et il ajoute, - nous retrouvons ici Marie de l'Incarnation -: «Je ne diray rien des Mères Ursulines; elles m'escrivent avec un tel feu et en si grand nombre, et de tant de divers endroits, que si on ouvroit la porte à leurs désirs, on composeroit une ville de religieuses et il se trouveroit dix maistresses pour une escholière... Le temps viendra que quelquesunes d'entre elles obtiendront ce que demande une armée. Nostre Seigneur en fera le choix. $\gg 10$

8 Introduction aux Annales de l'Hôtel-Dieu de Québec, 1636-1716, composées par Jeanne-Fr. Juchereau et Marie-A. Duplessis (Montréal, 1939), V. Déjà, en 1545, la traduction de l'appel de saint François Xavier aux étudiants de Sorbonne avait soulevé un gros émoi: Copie d'une lettre missive des Indes par Monsieur maistre François Xavier à son prevost Monsieur Egnace de Layola (Paris, 1545).

9 Relation de 1635 (éd. R. G. Thwaites, The Jesuit Relations (Cleveland, 1897), VII: 256; (éd. de Québec, 1858), I: 2). Cette même Relation expliquait en quoi consistait la «vocation spéciale» des missionnaires de la Nouvelle-France (Sentimens et advis, n. 30, éd. Thwaites, VIII: 188; éd. Québec, I: 49).

10 Relation de 1637 (éd. Thwaites, XI: 58; éd. de Québec, I: 5). Paul Le Jeune (1591-1664) s'était converti du protestantisme et était entré chez les Jésuites en 1613. Après avoir été supérieur de la petite résidence des Jésuites de Dieppe, - circonstance d'importance dans l'histoire de la Mission -, il fut mis à la tête de la Mission du Canada, $1632-1641$; il rentrera alors en France. Il rédigea les Relations annuelles de la Nouvelle-France des années 1632 à 1642 (cette dernière est aussi de lui). "Cet apôtre infatigable, constate dom Jamet, ne recula devant aucune expérience ni aucune aventure pour prêcher l'Evangile» (Ecrits.... II : 322). Marie de l'Incarnation semble être entrée en relations épistolaires avec Le Jeune dès 1636 (Ecrits ..., III : 94-96). 
Lorsque cette Relation paraît et circule, l'effervescence missionnaire est déjà considérable. Madame de La Peltrie échafaude son romanesque mariage avec Jean de Bernières pour être plus libre de disposer de ses biens et de sa personne en faveur du Canada. La duchesse d'Aiguillon vient d'accorder, le 15 août 1637, une donation aux hospitalières de Dieppe pour la fondation du futur Hôtel-Dieu de Québec. «Cette donation, précise-t-elle, est faite pour être ledit hôpital dédié à la mort et au précieux Sang du Fils de Dieu répandu pour faire miséricorde à tous les hommes, et pour luy demander qu'il l'applique... pour tous ces pauvres peuples barbares. $\gg 11$ Une des principales inspiratrices de la duchesse venait de mourir cette année-là. Madeleine de Saint-Joseph, la «première prieure française du premier monastère des carmélites déchaussées en France 》, "avait un zèle merveilleux, explique la duchesse d'Aiguillon, pour la dilatation du christianisme en la Nouvelle-France. ${ }^{12}$ L'oncle de Madeleine n'était autre que Noël Brûlart de Sillery qui fondait l'année même la «réduction» qui porta son nom. ${ }^{13}$ La «bienfaitrice inconnue » de Montréal, Madame Claude de Bullion, lui était apparentée. Jeanne Mance lui avait été présentée en janvier $1641 .^{14}$

11 J.-F. Juchereau et M.-A. Duplessis, Annales de l'Hôtel-Dieu de Québec, ouvr. cité, 9. Le texte complet dit: « ... qu'il l'applique sur l'âme de Monseigneur le cardinal duc de Richelieu et celle de Madame la duchesse d'Aiguillon, et pour...»

12 La Vénérable Madeleine de Saint-Joseph (1578-1637), (Clamart, 1937), 358.

13 Noël Brûlart de Sillery (1577-1640), commandeur de l'Ordre de Malte et ambassadeur de Louis XIII, ami de saint François de Sales, se convertit en 1625. Sous l'influence de saint Vincent de Paul, il est ordonné prêtre en 1634. Depuis sa conversion, sa vie est toute de charité. Voir M. Fosseyeux, La conversion du Commandeur Brûlart de Sillery, dans Bulletin de la Société de l'histoire de Paris ..., (1910), XXXVII: 184-202; Roger Graffin, Noël Brûlart de Sillery et la fondation de Sillery au Canada, dans Travaux de l'Académie nationale de Reims, t. 108, 18991900, II (1902) : 207-222; P. Coste, S. Vincent de Paul, Correspondance..., (Paris, 1925), XIV, Tables.

14 Angélique Faure, nièce de Noël Brûlart de Sillery (la mère d'Angélique était une Brûlart de Sillery), fut mariée en 1612 à Claude de Bullion, futur surintendant des finances. Son mari, mort en décembre 1640 , lui laisse une immense fortune, qu'elle distribue à profusion et à bon escient, mais avec un souci scrupuleux de l'anonymat. Jeanne Mance, mise en relations, dès la fin de janvier 1641, avec la dame de Bullion 
Mais l'appel missionnaire suscite un mouvement de plus en plus étendu dans le monde laïc. La Compagnie du Saint-Sacrement, fondée en 1630 par Henri de Lévis, duc de Ventadour, ${ }^{15}$ animée par le baron Henri de Renty ${ }^{16}$ et bien d'autres, ${ }^{17}$ s'intéresse à l'évangélisation du Canada.

Jérôme Le Royer de La Dauversière réorganise l'Hôtel-Dieu de La Flèche et met sur pied une congrégation d'hospitalières,

par le récollet Archange Rapine, se voit chargée de la fondation de l'Hôtel-Dieu de Montréal, que la surintendante dotera avec munificence. Confirmée dans sa vocation par les jésuites J.-B. de Saint-Jure et Charles Lalemant, Jeanne Mance s'embarque à La Rochelle en juin, après avoir convenu avec Jérôme Le Royer de La Dauversière du Dessein des Messieurs de Montréal. Madame de Bullion mourut en 1664. On retrouve l'essentiel de l'histoire de la «bienfaitrice inconnue» dans l'ouvrage de la Sœur Maria Mondoux, L'Hôtel-Dieu premier hôpital de Montréal (Montréal, 1942), qui a pour base les Annales de l'Hôtel-Dieu de Montréal de la Sœur Marie Morin $(+1730)$, publiées en 1921. Voir aussi: Marie-Claire Daveluy, «Madame Claude de Bullion (1593-1664)》, dans la Revue d'histoire de l'A mérique Française, IX (1955) : 141-149.

15 Voir Pierre-René Le Voyer d'Argenson, Annales de la Compagnie $d u$ Saint-Sacrement, publiées par H. Beauchet-Filleau (Marseille, 1900); E. Levesque et R. Heurtevent, art. "Compagnie du Saint-Sacrement 》, dans le Dictionnaire de spiritualité, II: col. 1301-1311. On sait que le duc de Ventadour avait acheté, en 1625, la charge de vice-roi de la NouvelleFrance à son oncle le duc de Montmorency et l'avait revendue en 1627 au cardinal de Richelieu. C'est sous la vice-royauté de Ventadour que cinq Jésuites passèrent (1625) au Canada, dont Charles Lalemant (1587-1674), Jean de Brébeuf (1593-1649) et Ennemond Massé (1574-1646).

16 Gaston de Renty (1611-1649), «humble serviteur de nos seigneurs les pauvres », marié en 1633, est dirigé par Charles de Condren et JeanBaptiste de Saint-Jure. Sa vie de charité et sa vie mystique en firent un directeur spirituel très écouté. Il s'intéressa à toutes les œuvres charitables de son temps; il est notamment l'âme de la Compagnie du Saint-Sacrement et l'un des membres les plus actifs de la Société de Montréal. Saint-Jure composa L'idée d'un chrestien parfait. La vie de Monsieur de Renty (Paris, 1651) ; voir aussi: A. Bessières, Deux grands méconnus ... Gaston de Renty et Henry Buch (Paris, 1931).

17 On n'oubliera pas l'esprit et l'influence des congrégations secrètes, ou des Aa, nées au sein des congrégations mariales. Apparues au collège de La Flèche en 1630/1632, elles essaimèrent assez rapidement. A celle du collège de Clermont, à Paris, appartinrent François de Montmorency-Laval, premier évêque de Québec, et Henri Boudon, dont les œuvres spirituelles furent très lues au Canada. Le zèle du Père Alexandre de Rhodes (15911660), de retour du Tonkin et de la Cochinchine, enthousiasma le cercle parisien. Sur les Congrégations secrètes, voir l'article de R. Rouquette, dans le Dictionnaire de spiritualité, II : col. 1491-1507.

Le collège de La Flèche fut un centre de rayonnement missionnaire, sans doute unique en France pendant la première moitié du $17 \mathrm{e}$ siècle; cf. C. de Rochemonteix, Le collège Henri IV de La Flèche (4 vol., Paris, 1889) et Les Jésuites et la Nouvelle-France au XVIIe siècle (3 vol., Paris, 1895-1896). 
qui fondera plus tard l'Hôtel-Dieu de Montréal. ${ }^{18}$ La "Compagnie des Messieurs et Dames de Montréal » reprend à son compte les intentions missionnaires, que les Cent-Associés laissaient plus ou moins en sommeil. Ces Messieurs expliquent au pape Urbain VIII, dans une supplique de 1643: «Déjà, depuis les années précédentes, les Associés ont fait passer dans cette île [de Montréal] jusqu'à soixante-dix hommes, tant nobles chevaliers qu'artisans; et présentement encore ils y envoient plusieurs autres pour travailler la terre, pour ouvrir aux barbares les portes de la foi et celles de la vie civile, pour donner aux sains et aux malades, dans l'hôpital qu'ils y élèvent, des aliments, des remèdes et des exemples de piété et de charité, afin de les y engendrer à Jésus-Christ . . . » 19

Il est superflu d'insister sur les visées essentiellement apostoliques des missionnaires. Les Relations en témoignent abondamment. Et si, d'aventure, leur genre «édifiant» était, sur ce point, sujet à caution, ouvrons les lettres personnelles. Nous nous souviendrons cependant de la remarque, exagérée d'ailleurs, de Claude Boucher, assistant des jésuites de France à Rome: «Les Relations ne disent que le bien et les lettres que le mal. » 20

18 Jérôme Le Royer de La Dauversière est né à La Flèche en 1597; il suit les cours du collège des Jésuites, où il est condisciple des futurs missionnaires du Canada: Charles Lalemant, Barthélemy Vimont, Anne de Noue, Paul Le Jeune, Paul Ragueneau. "Receveur des aydes et tailles en l'élection de La Flèche », il se maria en 1618 et eut cinq enfants. Jérôme est directement intéressé à la fondation, en 1636, de l'Institut des Sœurs Hospitalières de Saint-Joseph à La Flèche. Dévotion à la Sainte Famille, rencontre de Jean-Jacques Olier, inspirations intérieures, audace missionnaire, dévouement désintéressé de Jeanne Mance et de Maisonneuve, conduisent peu à peu Jérôme Le Royer à l'organisation de la Compagnie des Messieurs et Dames de Montréal. Après avoir soutenu la fondation et les premiers développements de Ville-Marie, au milieu de vicissitudes sans nombre, Jérôme meurt en 1659. L'Hôtel-Dieu, premier hôpital de Monttréal, de la Sœur Mondoux (Montréal, 1942) constitue une des meilleures biographies de Jérôme Le Royer; voir aussi: A. Jamet, "Jérôme Le Royer de La Dauversière et les commencements de Montréal», dans la Revue de l'Université d'Ottawa, VI (1936) : 387-419.

19 D'après le texte des Archives départementales de Versailles (Fonds des Récollets de Saint-Denis, 57, 1) et d'après E.-M. Faillon, Histoire de la colonie française en Canada (Villemarie, 1865), I: 469-470.

20 Lettre au P. Bagot, 27 août 1663. Claude Boucher continuait: «Les Relations ne doivent pas être lues avec ce préjugé qu'elles disent toutes choses, mais seulement ce qui est d'édification» (cité par C. de Rochemonteix, Les Jésuites et la Nouvelle-France au 17e siècle (Paris, 
La correspondance privée parvenue jusqu'à nous, moins apprêtée sans doute que les lettres officielles ou communes, manifestent une donation de soi sans réserve ni retour au service des sauvages. Saint Charles Garnier, qui sera tué le 7 décembre 1649, écrivait à son frère le 12 août précédent: "Je vous prie de demander à Notre-Seigneur deux choses pour moy: la première, que dans ce tracas je demeure uni à lui; la deuxième, que je ne mette empeschement à faire par moy ce qu'il luy plaist pour le salut de ces pauvres peuples. ${ }^{21}$ Ce texte, en sa discrétion, nous pourrions en citer mille autres semblables —, révèle l'âme, heureuse et abandonnée, des missionnaires, martyrs ou non.

Il est temps de revenir à Marie de l'Incarnation et à son sens missionnaire. ${ }^{22}$ Appelée en Nouvelle-France par des invi-

1905), I: XVI). Marie de l'Incarnation fait cette remarque, rapportée dans la Relation de 1642: \& Si on vouloit dire toutes les choses qui peuvent donner de l'édification dans les actions de nos filles ce ne seroit jamais faict \& (éd. Thwaites, XXII : 182; éd. de Québec, II : 32). Les historiens ont relevé ce point, par exemple: A. Jamet, dans son introduction aux Annales de l'Hôtel-Dieu de Québec, ouvr. cité, p. XLI, et Léon Pouliot, Etude sur les Relations des Jésuites de la Nouvelle-France (1632-1672), coll. des Studia Collegii Immaculatæ Conceptionis (Montréal-Paris, 1940), notamment le ch. $3^{\mathrm{e}}$ de la $1^{\mathrm{e}}$ partie: 40-70.

21 A son frère Henri de Saint-Joseph, carme. La plupart des lettres connues de Charles Garnier ont été publiées dans le Rapport de l'archiviste de la province de Québec pour 1929-1930 (Québec, 1930), 1-43. Plusieurs ont été rééditées par Fr. Roustang, Jésuites de la Nouvelle-France, coll. Christus, n. 6 (Paris, 1961): 285-314. On trouvera dans cette précieuse anthologie d'autres lettres des martyrs, de fait ou de désir.

22 Marie Guyart est née à Tours le 28 octobre 1599 d'honnêtes artisans. Son père, Florent, était maître boulanger; sa mère, Jeanne Michelet, s'apparentait aux Babou de la Bourdaisière. A 18 ans, en 1617, elle est mariée par ses parents à Joseph-Claude Martin, maître ouvrier en soierie. Claude, leur fils, naît le 2 avril 1619, mais le père meurt à la fin de cette même année. Après quelque temps, Marie Martin entre au service de sa sœur Claude et de son beau-frère Pierre Buisson; elle devient vers 1624-1625, la régisseuse de l'entreprise de transports des Buisson. Le 25 janvier 1631, Marie Martin est admise au monastère des Ursulines de Tours; elle reçoit le nom de Marie de l'Incarnation. Elle accoste au rivage de Québec le $1^{\text {er }}$ août 1639. Elle fonde le monastère - «le Vieux Monastère de Québec 》-; elle ouvre la première école pour les jeunes «sauvagesses »; elle soutient colons, soldats et missionnaires jusqu'à sa mort survenue le 30 avril 1672 .

La meilleure biographie de Marie de l'Incarnation reste toujours celle publiée par son fils en 1677, puis celle de F.-X. de Charlevoix parue en 1724 (voir note 4). A signaler aussi les Vies publiées par l'abbé Richaudeau en 1873 à Tournai, par Marie de Chantal (Paris, 1893; refondue en 1910), par le chanoine J.-L. Beaumier, Marie Guyart de l'Incarnation (3e éd., Trois-Rivières, 1959). La Mère Marie-Emmanuel a brossé un portrait de Marie de l'Incarnation d'après ses lettres (Québec-Ottawa, 1946). La meil- 
tations intérieures de plus en plus pressantes, Marie de l'Incarnation vit, travaille, prie, se sacrifie pour «l'amplification du royaume de Jésus-Christ $\gg .{ }^{23}$ C'est par cette expression favorite qu'elle exprime le désir de tout son être de participer activement à l'évangélisation. C'est sans doute le «neuvième état d'oraison 》 décrit en sa seconde Relation qu'il faudrait relire et commenter ligne à ligne pour saisir en sa profondeur le sens apostolique et missionnaire de Marie de l'Incarnation. Elle en raconte avec précision les origines et les premiers développements: le songe survenu pendant l'octave de Noël 1633 , où elle reconnaîtra plus tard la préfiguration de sa mission au Canada, - l'enseignement qu'elle donne aux jeunes religieuses, où elle pressent une préparation à un nouvel état de vie, ${ }^{24}$ - ce qu'enfin, elle appelle « une émanation de l'esprit apostolique» du Christ, en son propre esprit. Cet «esprit apostolique », précise-t-elle, l'entraînait aussi bien «dans les Indes, au Japon, dans l'Amérique, dans l'Orient, dans l'Occident... et dans toute la terre habitable où il y avait des âmes raisonnables que je voyais toutes appartenir à Jésus-Christ $\gg^{25}$

leure manière d'aborder la spiritualité de Marie de l'Incarnation sera toujours la lecture directe de ses écrits. On trouverait une bonne initiation soit dans la Vie publiée par Claude Martin, soit dans l'annotation d'Albert Jamet à l'édition critique. L'ouvrage de Paul Renaudin, Marie de l'Incarnation Essai de psychologie religieuse (Paris, 1935), demeure, en tout état de cause, une très utile introduction. L'esquisse d'Henri Bremond est des plus brillantes (Histoire littéraire du sentiment religieux en France (Paris, 1922), VI : 1-226). Consulter l'exposé méthodique et solide de Fernand Jetté, O.M.I., La voie de la sainteté d'après Marie de l'Incarnation (Ottawa, 1954). 23 Cette expression lui est familière, par exemple, $2^{\mathrm{e}}$ Relation, $9^{\mathrm{e}}$ état d'oraison, dans l'édition d'A. Jamet, Ecrits ..., II : 314 et 315 . Le mot est assez communément employé à cette époque; voir le texte des Véritables motifs, cité plus loin, ou encore Relation de 1642 (éd. Thwaites, XXII: 42; éd. de Québec, II : 4).

24 Dans ces leçons, Marie de l'Incarnation ne peut se retenir de laisser entrevoir son expérience de la foi ( Je ne pouvais me taire et il fallait que j'obéisse à l'Esprit qui me possédait 》) et le feu qui la consume pour le salut des âmes. Elle a de plus en plus l'impression qu'elle n'est au monastère de Tours que «comme en un lieu de refuge» où Dieu la «dispose pour ses desseins $\gg$, sans qu'elle puisse faire autre chose que de « s'abandonner entièrement à Dieu 》 (9e état d'oraison, éd. A. Jamet, Ecrits ..., II : 306-309). $259^{e}$ état d'oraison, ibidem, II: 310. Marie de l'Incarnation poursuit: « Je me promenais en esprit dans ces grandes vastitudes et j'y accompagnais les ouvriers de l'Evangile, auxquels je me sentais unie étroitement à cause qu'ils se consommaient [sic] pour les intérêts de mon céleste et divin Epoux, et il m'était avis que j'étais une même chose avec eux 》 (311). 
Ce sens universel de l'évangélisation restera toujours aussi vivace chez elle, même lorsqu'il se concrétisera et aura pour point d'application le Canada. ${ }^{26}$ "Je ne voyais plus d'autre pays pour moi que le Canada, dira-t-elle de ses années 1636-1639, et mes plus grandes courses étaient dans le pays des Hurons, pour y accompagner les ouvriers de l'Évangile. » ${ }^{27}$ Qui ne se souvient de la célèbre apostrophe à Madame de La Peltrie ? Lorsque Marie de l'Incarnation est mise au courant par le Père AntoineJoseph Poncet du dessein de la normande missionnaire, elle écrit à sa future compagne: "Quoi! Madame, notre divin Maître Jésus vous veut-il introduire dans le paradis terrestre de la Nouvelle-France ?... Il est vrai qu'il y a des glaçons, des ronces, des épines; mais le feu du Saint-Esprit a un souverain pouvoir pour consommer [sic] tout cela et même pour fendre les rochers. $\gg^{28}$

Que signifie donc «accompagner les ouvriers de l'Évangile »? Marie de l'Incarnation, pas plus que les autres mission313-319.

${ }^{26}$ C'est le dernier trait expliqué dans le 9e état d'oraison, ibidem:

27 Ibidem: 316. Marie de l'Incarnation répète presque immédiatement et insiste: «Je faisais bien des stations par tout le monde; mais les parties [les régions] du Canada étaient ma demeure et mon pays, mon esprit étant tellement hors de moi et abstrait du lieu où était mon corps..., que même en prenant ma réfection, c'étaient les mêmes fonctions et courses dans le pays des sauvages pour y travailler à leur conversion et aider les ouvriers de l'Evangile. Et les jours et les nuits se passaient de la sorte » (316-317).

28 Lettre de novembre 1638, éd. A. Jamet, Ecrits ..., III: 111. L'expression «paradis terrestre 》 vient sans doute des Relations, de celle de 1635 peut-être (éd. Thwaites, VIII: 170; éd. de Québec, I: 45). Madeleine de Chauvigny, née à Alençon en 1603, fut mariée en 1622 à Charles de Gruel de La Peltrie; devenue veuve en 1628, elle accompagnera Marie de l'Incarnation au Canada avec le titre de «fondatrice» du monastère des Ursulines de Québec. Sauf les deux années ou elle suivra Jeanne Mance à Montréal, elle habita tout à côté du monastère et s'adonna à une vie débordante d'activité. Elle mourut en novembre 1671, cinq mois avant Marie de l'Incarnation. Celle-ci raconta dans une lettre de Noël 1670 au Père AntoineJoseph Poncet la vocation de la «fondatrice 》; voir Lettres de ... Marie de l'Incarnation, éd. Richaudeau (Paris, 1876), II: 490-503. 
naires, hommes ou femmes, n'apporte ses biens ${ }^{29}$ à la mission, mais elle se donne elle-même pour servir. Servir est le mot humble et simple qu'emploient alors ceux et celles qu'attire l'obscur dévouement à l'évangélisation de la Nouvelle-France. Ce service, dans l'esprit de ces hommes et de ces femmes, inclut explicitement l'usure du corps dans l'anonyme besogne quotidienne, avec ses rudesses, ses humiliations, son épuisant recommencement en un pays où tant de choses rebutent humainement. Ce service inclut expressément aussi les croix de toute sorte, des plus menues aux plus insolites, il inclut surtout le désir véhément du martyre.

Comment dire et illustrer ce qui fut le mobile secret de tant de cœurs? Les confidences s'en font volontairement rares. Marie de l'Incarnation nous en avertit elle-même, ou plutôt elle en avertissait le Père Le Jeune qui lui avait demandé de la copie pour la Relation de 1642: "Suffit que Dieu, répond-elle avec fermeté, qui est nostre Père, scache avec quel amour nous servons nos néophytes; c'est assez que luy seul cognoisse ce qui se passe dans cette petite maison, sans qu'il soit produit aux yeux des hommes. » 30

Les attraits humains du service des jeunes «sauvagesses》 comme le soin des malades et des vieillards ne pouvaient être que de courte durée, on le pense bien. Le récit des premiers contacts des Ursulines avec leurs nouvelles élèves est sans doute charmant dans sa naïveté. Les yeux s'ouvrirent vite et l'acclimatation dut être parfois un supplice. ${ }^{31}$ Le Père Paul Le Jeune

${ }^{29}$ Les chrétiens et les chrétiennes de France qui ne partaient pas donnaient de leurs biens, et parfois fort généreusement, comme la nièce de Richelieu et ces « Messieurs et Dames de Montréal 》. Marie de l'Incarnation, entrée d'ailleurs sans dot chez les Ursulines, laissait son avoir à son fils Claude, et sa sœur, Claude Buisson, gérerait ce capital.

30 Relation de 1642 (éd. Thwaites, XXII : 182; éd. de Québec, II : 32 ).

31 Dès le 3 septembre 1640, Marie de l'Incarnation raconte à l'une de ses correspondantes comment sont reçues les jeunes pensionnaires: «Quand on nous les donne, elles sont nues comme un ver, et il les faut laver depuis la tête jusqu'aux pieds, à cause de la graisse dont leurs parents les oignent par tout le corps; et quelque diligence qu'on fasse et quoiqu'on les change souvent de linge et d'habits, on ne peut de longtemps les épuiser de la vermine causée par l'abondance de leurs graisses. Une sœur emploie une partie du jour à cela. C'est un office que chacune ambitionne avec empressement 》 (éd. A. Jamet, Ecrits ..., III: 177). Dans sa seconde Relation, Marie de 
constatait avec quelque humour dans la Relation de 1642: «Il faut avoir de bons yeux pour ne voir que Jésus-Christ dans les Sauvages... C'est un attrait dont je n'attens la persévérance que de Jésus-Christ mesme. ${ }^{32}$ Il en avait d'ailleurs prévenu ceux et celles que tenterait la Nouvelle-France au chapitre $12^{\mathrm{e}}$ de la Relation de 1634, qu'il intitulait: «De ce qu'il faut souffrir hivernant avec les Sauvages ${ }^{33}$ ». Saint Jean de Brébeuf donnera un Advertissement d'importance pour la mission huronne, dans la Relation de $1636 .^{34}$ Les Ursulines comme les Hospitalières avaient lu ces textes.

Aussi n'est-il pas étonnant que certains tempéraments, plus délicats ou plus mélancoliques, n'aient pu résister au dépaysement. D'autres, pour tenir, se sont livrés à Dieu dans un abandon sans partage. L'un des exemples les plus typiques est celui de l'hospitalière Catherine de Saint-Augustin. Arrivée au pays en 1648, âgée de seize ans, et tentée de retourner en France, elle s'oblige par vœu, le 18 octobre 1654, à rester. Cette page, qui mérite tout respect, doit être retenue.

Jésus-Christ, mon Sauveur, qui, par une disposition toute aimable de votre Providence divine, avez voulu me donner place en ce païs, quoy que j'en sois très indigne, je, sour Catherine de Saint-Augustin, désirant de tout mon cœur coopérer aux saintes inten-

l'Incarnation rappellera que, dans les premiers temps, «la saleté des filles sauvages... nous faisait quelquefois trouver un soulier en notre pot, et journellement des cheveux et des charbons, ce qui ne nous donnait aucun dégoût » (Ibidem, II : 372). On relèverait bien d'autres traits encore.

32 Relation de 1642 (éd. Thwaites, XXII : 170; éd. de Québec, II : 29-30). Le texte complet est celui-ci: «Je sçay bien que la vertu est aimable par tout, mais elle est plus agréable sous la panne [étoffe de soie, de laine...] et sous le satin, et dans des âmes et des corps bien polis, que sous des haillons et dans des personnes qui ne connoissent point d'incivilitez, pource qu'ils n'ont pas seulement les premiers principes de la civilité. Que les Hospitalières aiment constamment des malades et des pauvres, et les Ursulines des séminaristes et des femmes sauvages dans lesquels on ne void que Jésus-Christ tout pur, sans aucun attrait qui flatte les sens, c'est un attrait dont je n'attens la persévérance que de Jésus-Christ mesme. 》

33 (Ed. Thwaites, VII : 34-64; éd. de Québec, I: 51-57). Chapitre reproduit par F. Roustang, ouvr. cité, 49-60.

34 Advertissement d'importance pour ceux qu'il plairroit à Dieu d'appeller en la Nouvelle France et principalement au pays des Hurons (éd. Thwaites, X: 86-114; éd. de Québec, I: 92-99). Texte reproduit par F. Roustang, ouvr. cité, 109-120. 
tions qu'a sur moy votre saint amour, prosternée aux pieds de votre divine Majesté, en la présence de votre sacrée Mère, ma sainte Reine et Maîtresse, de son glorieux Epoux, mon bon ange gardien, mes saints patrons et de toute la cour céleste, fais vœu de perpétuelle stabilité en ce païs, entendant le tout selon la volonté de ceux qui me conduisent. Je vous conjure, mon adorable Sauveur, qu'il vous plaise me recevoir pour votre perpétuelle servante et esclave en ces contrées et me rendre digne d'une vocation si excellente. ${ }^{35}$

Servir, n'est-ce pas aussi la devise de ces deux grandes âmes, Jeanne Mance ${ }^{36}$ et Marguerite Bourgeoys ${ }^{37}$ qui se dévouèrent à Montréal, simples, obscures, pauvres, incomprises, au service des malades ou à l'instruction des enfants ? Toutes deux s'en retournèrent chacune trois fois au royaume de France pour y traiter des besoins particuliers de la Mission; elles revinrent au Canada y souffrir et y mourir.

Servir, coûte que coûte, par la Croix jusqu'au martyre.

Lorsque saint Isaac Jogues raconte à son supérieur, le 6 août 1643, sa longue et terrible captivité entre les mains des Iroquois, il termine par cette profession solennelle: «Bien que très probablement je pusse m'enfuir, si je le voulais, en passant soit chez les Européens, soit chez d'autres barbares nos voisins, cependant, sur cette croix, où le Seigneur m'a fixé avec lui, j'ai décidé de vivre et de mourir avec l'aide de sa grâce. »

35 Paul Ragueneau, La vie de la Mère Catherine de Saint-Augustin, religieuse hospitalière de la Miséricorde de Québec en la Nouvelle France (Paris, 1671), 83. Il ne serait pas surprenant que le texte cité s'inspirât d'un vœu semblable fait en 1647 par saint Noël Chabanel, qui sera massacré à la fin de 1649; voir ce texte dans Fr. Roustang, ouvr. cité, 320-321. Les deux formules sont fort proches l'une de l'autre. Ce vœu était-il connu et répandu ?

36 Marie-Claire Daveluy, Jeanne Mance, 2e éd., (Montréal, 1962). Le dossier en vue du procès de béatification constitué par Sœur Mondoux et M.-Cl. Daveluy met bien en relief le sens du « service » chez Jeanne Mance (1606-1673).

37 La bienheureuse Marguerite Bourgeoys (1620-1700) a eu son historien, dom Albert Jamet (2 vol., Montréal, 1942). La Vie de la Scur Marguerite Bourgeoys, dite du Saint-Sacrement, composé par Charles de Glandelet en 1701 et 1715 , retrace * le vrai esprit 》 de la fondatrice et de l'Institut de Notre-Dame; elle demeure l'une des sources essentielles. 
Jogues explique ensuite les raisons de sa détermination et du choix délibéré de la croix: «Qui, en effet, pourrait consoler en mon absence les Français prisonniers et absoudre ceux qui se confesseraient? Qui pourrait avertir de leur devoir les prisonniers hurons baptisés, instruire les nouveaux prisonniers qui sont périodiquement amenés, baptiser les mourants et les soutenir dans les tortures ? Qui pourrait régénérer les enfants dans les eaux sacrées, pourvoir au salut des adultes mourants et à l'instruction de ceux qui sont en santé ?» $\mathbf{3 8}$

Pas plus que les missionnaires, Marie de l'Incarnation n'a jamais séparé croix et martyre. Pour elle comme pour eux,et c'est un des traits qui les rapprochent de l'Église primitive -, être missionnaire, c'est être candidat au martyre, comme Brébeuf en fit le vœu. ${ }^{39}$ Marie de l'Incarnation n'avait guère besoin de la leçon qu'inspiraient à Paul Le Jeune, en 1642, les enthousiastes desseins des Messieurs de Montréal: «Ces Messieurs me permettront de leur dire en passant qu'on ne mène personne à JésusChrist que par la croix, que les desseins qu'on entreprend pour sa gloire en ce pays se conçoivent dedans les dépenses et dedans les peines, se poursuivent dedans les contrariétés, s'achèvent dedans la patience et se couronnent dedans la gloire. $\gg^{40}$

L'ursuline de Québec distingue avec raison les intérêts de la foi de tout autre intérêt. Elle mande à l'un de ses frères: «Ceux qui ne viennent au Canada que pour le temporel [pour

38 Texte latin dans $\mathrm{Ph}$. Alegambe, Mortes illustres et gesta eorum de Societate Jesu qui... confecti sunt (Rome, 1657), 632; traduction d'après F. Roustang, ouvr. cité, 237. Charles Garnier écrit à son frère carme le 20 juillet 1636: "Je vous conjure de m'obtenir un don d'oraison et un esprit de croix .... (Rapport de l'archiviste ... pour 1929-1930, ouvr. cité, 9, et dans F. Roustang, 291).

39 «Mon Sauveur Jésus, je vous fais vœu de ne jamais manquer de mon costé à la grâce du martyre, si par vostre infinie miséricorde vous me le présentez quelque jour, à moy vostre indigne serviteur... Faites que je vive en telle façon qu'enfin vous m'octroyiez cette faveur de mourir si heureusement $\gg$ (texte latin et français dans la Relation de 1649, éd. Thwaites, XXXIV : 164-166; éd. de Québec, II : 18-19).

40 Relation de 1642 (éd. Thwaites, XXII: 210; éd. de Québec, II:37). Il faudrait méditer les Divers sentiments et advis des Pères qui sont en la Nouvelle France, recueillis par Paul Le Jeune dans la Relation de 1635 (éd. Thwaites, VIII: 168-192; éd. de Québec, I: 45-50) et dans F. Roustang, ouvr. cité, 72-82. "Une fois qu'on a gousté à bon escient la douceur de la croix de Jésus-Christ, on la préfère à tous les empires de la terre» (n. 5). 
le profit] n'y trouveront jamais leur compte si bien [aussi bien] que ceux qui y viennent pour donner leur vie pour Jésus-Christ. Si ceux-ci y souffrent, c'est de ne pas assez souffrir. » ${ }^{41}$ Il y a, en vérité, chez elle, un attrait vers la souffrance, - où, soit dit en passant, les psychanalistes seraient bien mal venus à chercher quelque morbidité, y a-t-il beaucoup de personnes aussi équilibrées que Marie de l'Incarnation ? - , qui est un attrait de conformité au Christ souffrant. "Aussi est-ce un paradis terrestre, - le Canada -, où les croix et les épines naissent si amoureusement que plus on en est piquée, plus le cœur est rempli de douceur. Priez Notre-Seigneur, confie-t-elle à une visitandine, qu'il me fasse la grâce de les aimer toujours. $\gg 42$

En «ce païs de croix $\gg,{ }^{43}$ comme l'appelaient les trois premières Hospitalières de Dieppe choisies pour l'Hôtel-Dieu de Québec, tous ceux qui ont quelque ferveur pour l'évangélisation ne peuvent se rassasier de croix. "Je me trouvay dans une avidité extrême des croix », notera Catherine de Saint-Augustin pendant ses exercices spirituels de 1666 ; et l'année suivante: «J'ay été portée d'un ardent et véhément désir de souffrir, en sorte qu'il m'a semblé... que je ne pourrais vivre sans croix. $\gg^{\mathbf{4 4}}$

La vraie croix, désirée de ceux et celles dont nous parlons, c'est de souffrir, de verser son sang et de mourir pour le Christ. Invinciblement, en lisant les textes spirituels de la NouvelleFrance au $17^{\mathrm{e}}$ siècle, on évoque les martyrs d'autrefois, ceux du Colisée, ceux de l'amphithéâtre de Lyon, de Smyrne ou de Carthage. "Je suis en une consolation très sensible du bon souhait que vous faites pour moi, le martyre, répond Marie de l'Incarnation à son fils Claude le 4 septembre 1641. Hélas ! mon très

41 Lettre du 4 septembre 1640 à son frère Hélye, Ecrits..., éd. A. Jamet, III : 184-185; relire surtout la lettre d'adieu à ce même frère, 15 avril 1639, ibidem, III : 126-128.

42 Lettre du 4 septembre 1640 à Marie-Gillette Roland, visitandine de Tours, Ecrits ...., III : 194.

43 J.-F. Juchereau et M.-A. Duplessis, Annales de l'Hôtel-Dieu de Québec, ouvr. cité, 12. Déjà, la Relation de 1634 disait des Pères Antoine Daniel et Jean de Brébeuf, futurs martyrs, "leur désir d'entrer dans le paīs de la Croix 》 (éd. Thwaites, VII: 218; éd. de Québec, I: 89).

$44 \mathrm{P}$. Ragueneau, La Vie de la Mère Catherine de Saint-Augustin, ouvr. cité, 124 et 268. 
cher fils ..., je n'ai rien fait jusques icy qui soit capable d'avoir gagné le cœur de Dieu, car, pensez-vous, il faut avoir beaucoup travaillé pour être trouvée digne de répandre son sang pour Jésus-Christ. » Et aussitôt cette mère étonnante a ce cri de foi qui ne peut surprendre un authentique chrétien: «Si on me venait dire: Votre fils est martyr, je pense que j'en mourrais de joie. $\gg 45$

\section{LAIICAT}

Une des données les plus neuves et les plus intéressantes de l'histoire religieuse et spirituelle de la Nouvelle-France au milieu du $17^{\mathrm{e}}$ siècle est l'importance prise par les laïques, qui soutiennent, organisent l'implantation coloniale et missionnaire et lui donnent un esprit.

Il convient de rappeler que c'est tout d'abord à des auxiliaires laïcs que les missionnaires ont offert d'ouvrir, à Québec, des « séminaires» pour les garçons et les filles des «sauvages » ou de tenir des hôpitaux. Un an à peine après la disparition des corsaires anglais, Paul Le Jeune faisait déjà appel à «quelque brave maistresse que le zèle de la gloire de Dieu et l'affection au salut de ces peuples fera passer icy, avec quelques compagnes animées de pareil courage $\gg .^{46}$ Madame de La Peltrie sera, à Québec, l'une de ces précieuses auxiliaires laïques. Mais la question du laïcat missionnaire est posée avant tout à Ville-Marie.

Je n'ai nullement l'intention de traiter des origines de la cité de Montréal, lorsque tant d'historiens et de biographes l'ont fait avec compétence. Je me permettrai seulement d'évoquer l'un ou l'autre document contemporain des événements qui ne laisse aucune ombre sur les intentions des principaux protagonistes, Jérôme Le Royer de La Dauversière et la Société de Montréal, animée par Gaston de Renty et Jean-Jacques Olier,

\footnotetext{
45 Ecrits ..., éd. A. Jamet, III : 232. Ce n'était point là souhait sans conséquence. Que de fois Québec fût-il sous la menace des Iroquois ! C'est encore à son fils qu'en 1650 elle mande: « Réjouissez-vous donc si nous mourons et si l'on vous porte la nouvelle que notre sang et nos cendres sont mêlés avec les leurs [ceux des chrétiens]. Il y a de l'apparence que cela arrivera, si les mille Iroquois qui se sont détachés pour aller à la Nation Neutre viennent rejoindre ceux qui sont à nos portes 》 (lettre du 30 août 1650, ibidem, IV : 287).
}

46 Relation de 1634 (éd. Thwaites, VI: 150-152; éd. de Québec, I: 12). 
d'une part, et d'autre part Jeanne Mance et Paul de Chomedey de Maisonneuve.

Trois documents paraissent essentiels pour saisir ces intentions: le Dessein des Messieurs de Montréal, la supplique que ces Messieurs adressèrent en 1643 au pape Urbain VIII - déjà mentionnée -, et la brochure sur les Véritables motifs de Messieurs et Dames de la Société de Notre-Dame de Montréal pour la conversion des sauvages de la Nouvelle-France, publiée la même année. Il est aisé de dégager, même à la suite d'une lecture rapide, les préoccupations majeures de ces Messieurs.

La Relation de 1642 consacre son chapitre $9^{\mathrm{e}}$ au Dessein des Messieurs de Montréal et donne, d'après un témoin, le récit, devenu classique, des événements et des intentions des personnages:

Environ trente-cinq personnes de condition se sont unies pour travailler à la conversion des pauvres sauvages de la Nouvelle France, et pour tascher d'en assembler bon nombre dedans l'Isle de Montréal qu'ils ont choisie, estimans qu'elle est propre pour cela; leur dessein est de leur faire bastir des maisons pour les loger, et défricher de la terre pour les nourrir, et d'établir des Séminaires pour les instruire et un Hostel-Dieu pour secourir leurs malades.

Tous ces Messieurs et Dames s'assemblèrent un jeudy vers la fin du mois de février de cette année 1642, sur les dix heures du matin en l'Eglise de Nostre-Dame de Paris, devant l'Autel de la Saincte Vierge, où un Prestre d'entr'eux dist la saincte Messe et communia les associez qui ne portent point le caractère [sacerdotal]. Ceux qui le portent célébrèrent aux autels qui sont à l'entour de celuy de la Saincte Vierge; là tous ensemble ils consacrèrent l'isle de Montréal à la Saincte Famille de Nostre Seigneur, Jésus, Marie et Joseph, sous la protection particulière de la Saincte Vierge; ils se consacrèrent eux-mêmes et s'unirent en participation de prières et de mérites, afin qu'estans conduits d'un mesme esprit, ils travaillent plus purement pour la gloire de Dieu et pour le salut de ces pauvres peuples, et que les prières qu'ils feront pour leur conversion et 
pour la sanctification d'un chacun desdits associez soient plus agréables à sa divine Majesté. ${ }^{47}$

Si le Dessein des Messieurs de Montréal s'harmonise parfaitement avec les intentions apostoliques des missionnaires, on trouverait sans doute difficilement plus belle apologie de l'apostolat des laïcs que celle exposée par l'auteur anonyme des Véritables motifs. Le missionnaire a sa fonction, irremplaçable; à côté de lui, le laïc a aussi sa mission. "Les laiques néanmoins, comme espousez de Jésus-Christ, enrôlés par le saint baptême en sa milice, sont obligez en cette qualité de rechercher à lui plaire, et, pendant que les autres [les missionnaires] sont avant dans la mêlée..., assister les combattants, chacun de ce qu'il peut et sait faire. $\gg 48$

L'auteur en appelle à l'exemple du Christ et de la primitive Église. «Après le martyre, (les laïques) ne tenaient rien tant à honneur que d'estre employés par les Pères de l'Église en quelque mission laborieuse, périlleuse et de dépense extraordinaire, comme d'estre envoyés en France, Espagne, Allemagne et autres pays infidèles en ces temps-là, où les uns seraient en personne pendant que les autres à Rome avaient la conduite et le soin des choses qui leur étaient nécessaires. $\gg 49$

Puisque le «saint désir d'amplifier le service de Dieu doit être aussi naturel à l'homme que le boire et le manger », et puisqu' «il n'est pas raisonnable de voir, les bras croisés et avec

47 Relation de 1642 (éd. Thwaites, XXII: 208-210; éd. de Québec, II: 37). Ce texte, adressé à un destinataire inconnu, se terminait par une invitation pressante: «Nous espérons tous que vostre Révérence embrassera cet ouvrage et qu'elle ira en personne aider ces pauvres infidelles pour leur faire connoistre leur Créateur. 》

48 Les Véritables motifs de Messieurs et Dames de la Société de Notre-Dame de Montréal (Paris, 1643), réédités par H.-A. Verreau dans la collection des Mémoires de la Société historique de Montréal, 9e livraison (Montréal, 1880) : 2.

49 Les Véritables motifs, ouvr. cité, 4. Un rapprochement s'impose avec un des avis (n. 18) de la Relation de 1635: "La nation des Hurons se dispose à recevoir la lumière de l'Evangile ...; mais il y faut deux sortes de personnes pour bien faire cela: les uns, de l'ancienne France, assistant de leurs sainctes prières et de leur charité; les autres, en la Nouvelle, travaillant avec grande douceur et infatigabilité de la bonté de Dieu; et de ce doux concert dépend la conversion de plusieurs milliers d'âmes, pour chacune desquelles Jésus-Christ a versé tout son précieux sang 》 (éd. Thwaites, VIII : 180 ; éd. de Québec, I : 47). 
des yeux tout secs, les mains percées et douleurs que notre époux et capitaine a employées à la conquête du monde », il importe à tous de «compatir avec lui », en « coopérant ce que chacun peut et doit faire », comme «autant d'abeilles ingénieuses à inventer les moyens d'estendre l'empire de cette saincte communauté à la gloire de son maître $\gg^{50}$

Qne ne pouvons-nous continuer la lecture des Véritables motifs! Nous y apprendrions comment on spiritualise ses actions, ce qu'est « l'esprit de la charité désintéressée », le service en dehors de «mercenaireté ». Nous retiendrions qu'il « ne faut pas ... estimer que Dieu nous ait ouvert des chemins auparavant inconnus à travers tant de mers pour en rapporter des castors et des pelleteries $\gg .{ }^{51}$

Les communautés chrétiennes en Nouvelle-France, si éphémères fussent-elles parfois, sont nées dans ce climat de foi et de générosité. La vie chrétienne des sauvages nous est contée par les Relations, les chroniques ou Annales des Hôtels-Dieu et des monastères, et aussi par la correspondance privée. En lisant tous ces textes, on constituerait sans peine un florilège abondant qui illustrerait la simplicité des sauvages baptisés et leur docilité à se laisser conduire dans la foi. ${ }^{52}$ Marie de l'Incarnation en sera toujours dans l'émerveillement. Leur goût de la prière, leur joie à chanter Dieu, leur assiduité à fréquenter les sacrements et à observer les pénitences publiques, «leur zèle pour la deffence et pour l'amplification de la foy $\gg,{ }^{53}$ leur confiance dans

50 Les Véritables motifs, ouvr. cité, 5 et 6.

51 Les Véritables motifs, ouvr. cité, 54, 56 et 42.

52 La simplicité et la docilité sont les deux qualités que relève le plus ordinairement Marie de l'Incarnation chez les «néophytes》, par exemple, Ecrits ...., éd. A. Jamet, III: 185-187.

53 L'expression est de Paul Le Jeune dans la Relation de 1642, (éd. Thwaites, XXII : 42; éd. de Québec, II : 4). Ce sens apostolique des nouveaux chrétiens jette dans l'admiration Marie de l'Incarnation et Paul Le Jeune lui-même. Charles de Tadoussac, «qui a été baptisé depuis peu, raconte l'ursuline à son ancienne supérieure, a plus fait par ses sermons que cent prédicateurs n'auraient fait en plusieurs années ... L L'on voyait prêcher deux apôtres en même temps, l'un jésuite, et l'autre sauvage, chrétien seulement depuis six mois 》 (lettre du 24 août 1641, Ecrits ..., éd. A. Jamet, III: 209). 
les missionnaires, en un mot leur ferveur prouvait à l'évidence qu'une élite chrétienne se formait peu à peu et se haussait même parfois jusqu'à l'héroïsme. Les missionnaires n'hésiteront pas, à l'occasion, à parler d'Église primitive. Les Véritables motifs décrivent les premières ébauches de la communauté chrétienne montréalaise, où «tous vivent en Jésus-Christ en un cœur et une âme, représentant en quelque façon la forme de la primitive Église $\gg .{ }^{54}$

Jeanne Mance et Maisonneuve ou surtout les Jésuites auraient-ils approuvé ce tableau idyllique d'une chrétienté naissante et difficile? Rien de moins sûr. C'est bien pourtant vers la réalisation d'une Église à l'image de l'Église primitive qu'ils tendent au milieu des dangers de toute sorte.

A Québec, où la chrétienté est plus ancienne, Marie de l'Incarnation revient sans cesse sur la foi des «néophytes》, adolescents et adultes. Sa lettre du 29 septembre 1642 à la supérieure des Ursulines de Tours n'est qu'un long récit d'action de grâces. "Nous voyons des sentiments si chrétiens dans nos bons néophytes que notre extérieur fait connaître la joie de nos cœurs; ce sont des biens du Paradis qui adoucissent les épines du Canada et les rendent plus aimables que tous les plaisirs de la terre. $\gg^{55}$ La vie communautaire à la «réduction» de Sillery et la vie

De Joseph Chihouatenhoua, merveilleux apôtre également, Marie écrira: «Voilà les sentiments d'un homme sauvage, mais que la grâce a poli au-delà de tout ce que je vous en pourrais dire » (lettre à la supérieure de la Visitation de Tours, 4 septembre 1660, ibidem, III: 187).

Le Père Le Jeune est plus vert! «Eussay-je jamais creu que des barbares nés dans la cruauté, nourris de chair humaine, fussent devenus prédicateurs de Jésus-Christ? Je puis assurer que je ne sçache personne qui leur ayt donné ces pensées d'aller inviter les autres nations de croire en Dieu; c'est un pur ouvrage du Sainct-Esprit» (Relation de 1640, éd. Thwaites, XVIII : 110 ; éd. de Québec, I: 11).

54 Les Véritables motifs, ouvr. cité, 20. Marie de l'Incarnation emploie fréquemment cette comparaison. Les missionnaires, dira-t-elle, « sont infatigables à cultiver nos bons chrétiens, qui vivent dans la perfection où vivaient ceux de la primitive Eglise 》 (lettre à son frère Hélye, 4 septembre 1640, Ecrits ..., éd. A. Jamet, III : 183-184). "Nous voyons dans notre primitive Eglise le zèle et l'ardeur de la primitive Eglise convertie par les Apôtres 》 (lettre à la supérieure de la Visitation de Tours, 4 septembre 1640 , ibidem, 186). \& Quant aux sauvages sédentaires, ils sont dans la ferveur des premiers chrétiens de l'Eglise 》 (lettre à son ancienne supérieure, 13 septembre 1640, ibidem, 207).

55 Ecrits ..., éd. A. Jamet, III : 273-296; la citation est à la p. 284. 
personnelle de Joseph Chihouatenhoua, ${ }^{56}$ sans compter l'exemple magnifique de Catherine Tekakwitha, ${ }^{57}$ sont, si l'on ose leur appliquer une expression de Jean de Brébeuf, de beaux «begayemens »d' « enfans spirituels ${ }^{58}$

Nous n'avons pas à insister ici sur la vie chrétienne des colons. Le rayonnement spirituel et apostolique des congrégations mariales témoigne pour eux. ${ }^{59}$ Par ailleurs, l'exemple de familles chrétiennes pourrait être allégué, depuis la famille de Louis Hébert jusqu'à celle de Jean Bourdon. Marie de l'Incarnation fait à son fils l'éloge et de Monsieur Bourdon, qui « avait avec moi une liaison de biens spirituels très particulière », et de sa seconde femme, qui est «la mère des misérables ${ }^{60}$ La vie chrétienne de Maisonneuve, gouverneur de Ville-Marie, a été profonde, exemplaire. ${ }^{61}$

56 Joseph Chihouatenhoua fut baptisé le 16 août 1637 par Jean de Brébeuf. Marié et père de famille, il exerce un apostolat extraordinaire dans sa tribu; sa vie chrétienne exemplaire est d'ailleurs un attrait considérable. Il fut massacré par les Iroquois le 2 août 1640. Les Relations ont parlé de lui à diverses reprises. Tous les textes qui témoignent de cette précieuse vie ont été délicatement présentés par Léon Pouliot, S.J., Le premier retraitant du Canada: Joseph Chihouatenhoua, Huron (Montréal, 1958).

57 Catherine Tekakwitha, «la sainte sauvagesse », née en 1656 dans la tribu des Agniers, fut baptisée par le P. Jacques de Lamberville, le jour de Pâques 1676 au village de Caughnawaga. Sa vie fut extraordinairement droite et pure. Elle mourut le mercredi saint 17 avril 1680. La Vie de Catherine Tegakouïta fut écrite par le P. Claude Chauchetière, en 1685; elle est reproduite dans le procès de béatification, Positio super introductione causæ, (Rome, 1938), 37-126.

58 Relation de 1636 (éd. Thwaites, X: 22; éd. de Québec, I: 79).

59 Voir, en particulier, l'article du P. Adrien Pouliot, cité à la note 80 .

${ }^{60}$ Lettre à Claude Martin, 1668, Lettres ..., éd. Richaudeau, II : 403405. Monsieur Boudon, confie-t-elle, \& avait une continuelle présence de Dieu et union avec sa divine Majesté $\gg$.

61 Paul de Chomedey de Maisonneuve, né en 1612, fut le fondateur de Ville-Marie et son gouverneur jusqu'en 1664; il sauva plus d'une fois la colonie de l'extermination. Il fut le plus ferme soutien de Jeanne Mance et de Marguerite Bourgeoys. Tous les annalistes parlent de lui avec de très grands éloges (Sœur Morin, Dollier de Casson, Sour Juchereau, etc.). "Cet amant de la très sainte Vierge... vécut à Montréal comme le père et le protecteur du peuple qu'il gouvernoit, recevant chez luy tous ceux qui n'avoient point d'azile, les aidant au delà de ce qu'ils osoient en attendre... Son désintéressement étoit si parfait qu'il ne s'est jamais aproprié la moindre chose des présents considérables que les sauvages luy faisoient... Il s'acquit l'estime de tout le païs 》 (Annales de l'Hôtel-Dieu de Québec, ouvr. cité, 110-111). Voir toute l'histoire de Maisonneuve dans A. Jamet, Marguerite Bourgeoys, ouvr. cité, passim. 
Personne n'ignore que cette élite, indienne ou française, n'est-ce pas le cas général ? - demeura un fait exceptionnel. Les exigences de la morale chrétienne, que les missionnaires ne minimisaient certes pas, écartaient le grand nombre. Aussi, dans la mentalité des fidèles, bien des petits côtés nous choqueraient aujourd'hui. Le haut fait d'armes de certains Algonquins «néophytes », tel que la Relation de 1642 le raconte, ne peut que nous laisser rêveurs sur la profondeur des sentiments chrétiens. Ils se ruent, dit l'auteur, sur les Iroquois, "tuent ceux qu'ils ont à la rencontre et, se voyant maistres de leurs corps et de leur bagage, se jettent à deux genoux par terre, rendent grâce à Dieu de la victoire, enlèvent les despoüilles et la chevelure de leurs ennemis vaincus et s'en reviennent triomphans à Sainct Joseph, visitant la maison de Dieu devant que d'entrer dans leurs cabanes. » ${ }^{62}$ Marie de l'Incarnation elle-même et tout son entourage, probablement, présenteront les projets militaires du gouverneur de Tracy comme une croisade: «Ce qui les anime tous, dit-elle des soldats, c'est qu'ils vont à une guerre sainte et qu'ils vont combattre pour la foi. $»^{63}$

En Nouvelle-France, comme ailleurs, au $17^{\mathrm{e}}$ siècle, on flaire partout l'intervention diabolique, on croit aux sortilèges, aux vengeances de Dieu. L'aventure des Ursulines de Loudun, si anormale qu'elle paraisse à nos mentalités du $20^{\circ}$ siècle, trouve créance, et, peut-être même, provoque-t-elle quelque mimétisme. Des personnes aussi lucides que Marie de l'Incarnation, Jérôme Lalemant, Paul Ragueneau, ont cédé à la tentation de voir dans l'extraordinaire tremblement de terre de 1663 une intervention maléfique des démons. ${ }^{64}$ En 1668, Marie de l'Incarnation accusera «la malignité de certains magiciens et sorciers venus de France » d'avoir infesté de démons une pauvre fille, sans doute

62 Relation de 1642 (éd. Thwaites, XXII : 54; éd. de Québec, II : 6). II : 290$)$.

63 Lettre à Claude Martin, 28 juillet 1665 (Lettres ..., éd. Richaudeau,

64 Marie de l'Incarnation raconte par le menu l'événement à son fils, lettre du 20 août 1663 (Lettres ..., éd. Richaudeau, II: 226-244). Les prémonitions de Catherine de Saint-Augustin en constituent le préambule ( $P$. Ragueneau, La vie de Mère Catherine..., ouvr. cité, 236-240). "Dieu ne se fâche contre nous, conclut l'ursuline, que pour nous sauver $\gg$ (Lettres..., 243). 
épileptique. ${ }^{65}$ Dès les années 1660 , la colonie se peuple et s'organise rapidement. L'ordre et les bonnes mœurs sont de plus en plus difficiles à garder. En certaines lettres de ses dernières années, apparaît chez Marie de l'Incarnation un certain désenchantement. Écrivant à son fils, en octobre 1669, elle se montre fort sévère sur les conséquences du trafic de l'eau-de-vie, «impuretés, viols, larcins, meurtres »; elle n'hésite pas à stigmatiser l'origine des maux de la colonie: «Il est vrai qu'il vient ici beaucoup de monde de France et que le pays se peuple beaucoup. Mais parmi les honnêtes gens il vient beaucoup de canaille de l'un et de l'autre sexe, qui causent beaucoup de scandale. » Aussi conclut-elle hardiment: «Il eût été bien plus avantageux à cette nouvelle Église d'avoir peu de bons chrétiens que d'en avoir un grand nombre qui nous cause tant de trouble. ${ }^{66}$ Ces difficultés grandissantes n'altèrent en rien son sain équilibre et sa foi robuste.

\section{DEVOTIONS, CHARISMES ET VIE CONTEMPLATIVE}

Ce qui soutenait, en toute leur vie, les missionnaires, ce qui soutenait Marie de l'Incarnation, ce fut leur attachement passionné au Christ, ils acceptaient avec enthousiasme de mourir pour lui et comme lui. De quelle manière se manifestait dans leur vie spirituelle journalière cet attachement passionné au Christ ? Si nous pénétrons quelque peu dans la vie chrétienne et spirituelle de la chrétienté canadienne, nous rencontrons nombre de dévotions, - d'ailleurs toujours vivaces dans le pays -, et nous remarquons la présence et l'influence de charismes, qui rappellent invinciblement le temps privilégié de la primitive Église. Ces dévotions et ces charismes apparaissent et se déve-

65 L'histoire remonte à plusieurs années, car il s'agit d'une pauvre malade, confiée à l'Hôtel-Dieu et donnée en garde à Catherine de SaintAugustin. Il semblerait que Catherine ait demandé à Dieu d'être molestée à la place de la malheureuse. Dans sa lettre à Claude Martin, 7 septembre 1668, Marie de l'Incarnation fait un bel éloge de la vie de charité de l'hospitalière (Lettres ...., éd. Richaudeau, II: 377-380).

66 Lettre à Claude Martin, octobre 1669 (Lettres ..., éd. Richaudeau, II : 436-437). Cette lettre sur les difficultés sociales et religieuses de la colonie est fort instructive. Marie de l'Incarnation tenait déjà pareil langage à son fils le 7 septembre 1668 , « parmi les honnêtes gens il nous vient de terrible racaille $\gg$ (Lettres ..., éd. Richaudeau, II : 377 ). 
loppent dans un climat de vie contemplative. Les dévotions soutiennent la foi et enserrent la vie chrétienne d'un réseau de pratiques religieuses vivantes, que l'accoutumance n'a pas encore défraîchies. Elles conduisent à Dieu et concrétisent le service de Dieu. Il s'agit de préférence de la dévotion à saint Joseph et à la sainte Famille, de la dévotion à l'Immaculée-Conception et au Cœur de Jésus.

Les Récollets consacrèrent le Canada à saint Joseph dès 1624. La Relation de 1637 décrit fastueusement «la feste du glorieux Patriarche sainct Joseph, Père, Patron et Protecteur de la Nouvelle France, l'une des grandes solemnitez de ce pays $\gg{ }^{67}$ Que de néophytes, que de chapelles et de paroisses seront mis sous la protection de saint Joseph ! C'est la dévotion des Récollets, comme des Jésuites et des Sulpiciens, celle des Ursulines comme celle des Hospitalières et des Filles de Saint-Joseph, fondées à La Flèche par La Dauversière en 1636 et qui essaiment à Ville-Marie en 1659. Saint Joseph est «le Patron du païs et protecteur de cette Église naissante ${ }^{68}$ «le Patriarche des Hurons », ${ }^{69}$ le Patron de la «Résidence de Saint-Joseph de Sillery », du «Séminaire Saint-Joseph » ou de la première école de sauvagesses, ouverte à Québec par Marie de l'Incarnation, le «Préfet perpétuel» de la congrégation mariale de Québec. ${ }^{70}$ L'hôpital de Montréal est fondé « au nom et en l'honneur de saint Joseph ${ }^{71}$ et la chapelle de l'hôpital lui est consacrée. ${ }^{72}$

67 Relation de 1637 (éd. Thwaites, XI: 66-70; éd. de Québec, I: 7-9). La première solennité de la fête de saint Joseph à Ville Marie eut lieu le 19 mars 1643; l'éclat en fut aussi grand qu'il était possible (voir Fr. Dollier de Casson, Histoire de Montréal. 1640-1672 (Montréal, 1871), 24). Les développements de L'Extraordinaire dévotion de la Nouvelle-France envers saint Joseph ont été présentés par Adrien Pouliot au Congrès d'études tenu à l'Oratoire Saint--Joseph à Montréal en 1955, Actes, Section historique (Montréal-Paris, 1956), 361-416, et par la Sœur M. Mondoux, La dévotion à saint Joseph à l'Hôtel-Dieu de Montréal, ibidem, 417-470.

68 Lettre du récollet Joseph Le Caron, en 1624, au grand vicaire de Pontoise, citée par Ch. Le Clercq, Premier etablissement de la foy dans la Nouvelle-France (Paris, 1691), I: 287.

69 D'après Jean de Brébeuf, Relation de 1635 (éd. Thwaites, VIII : 72; éd. de Québec, I : 24).

70 Acte de consécration du 19 mars 1664, d'après le Manuel des congréganistes de la Haute-Ville de Québec.

71 Contrat de fondation du 12 janvier 1644, signé par Claude de Bullion.

72 La première chapelle, exiguë, fut construite en 1645; la seconde, plus vaste, qui la remplace en 1658, servit d'église paroissiale. 
On ne peut séparer de la dévotion à saint Joseph celle à la sainte Famille, en raison même de ses origines. Les "Messieurs et Dames de Montréal » sont pour beaucoup dans sa diffusion. Le Royer de La Dauversière peut en être considéré comme le père. C'est lui qui, après s'être consacré, le 2 février 1630, avec sa femme et ses enfants, à la sainte Famille, fit approuver, dès 1636, par l'évêque d'Angers, une confrérie, formée dès 1634, en l'honneur de la sainte Famille, ou plutôt, selon l'ordonnance de Monseigneur Claude de Rueil, «sous le nom du glorieux saint Joseph, pour honorer à perpétuité la sainte Famille de JésusChrist... et parvenir à une parfaite adoration et glorification de l'auguste Trinité $\gg .{ }^{73}$

Cette dévotion envahit à ce point la vie spirituelle de Le Royer et imprégna si profondément ses entreprises qu'il donna aux membres de sa congrégation des Hospitalières de La Flèche le titre de Filles de Saint-Joseph et intitula leurs règles, en 1643, Constitutions de la communauté des Filles de Saint-Joseph, établies dans l'Hôtel-Dieu de La Flèche en l'honneur de la sainte Famille de nostre Seigneur. ${ }^{74}$ Mais déjà, le jeudi 27 février 1642, Ville-Marie avait été consacrée à la sainte Famille. ${ }^{75}$ Depuis leur arrivée, Jeanne Mance et Paul de Maisonneuve répandaient la dévotion. Rapidement elle devint populaire. ${ }^{76}$ Marie de l'Incarnation signale plusieurs fois que de nombreux soldats l'ont adoptée. «C'est notre grande dévotion ». ${ }^{77} \mathrm{Mgr}$ de Montmorency-

73 Le document daté du 17 février 1636, et rédigé en latin, se trouve aux Archives municipales du Mans, H. 1803; il est d'autant plus précieux qu'il semble bien autoriser la première confrérie de la sainte Famille, actuellement connue.

${ }^{74} \mathrm{La}$ Sœur M. Mondoux a relevé les principaux textes des Constitutions qui parlent de saint Joseph et de la sainte Famille; voir La dévotion à saint Joseph ..., art. cité, 431-435.

75 Voir Sœur M. Mondoux, L'Hôtel-Dieu premier hôpital de Montréal, 1642-1942 (Montréal, 1942), passim. Le récit en a été donné ci-dessus.

76 Maisonneuve créa même parmi les soldats, en 1663, une Milice de la Sainte-Famille, chargée d'assurer la sécurité de Ville-Marie.

77 Par exemple, lettres à son fils, 30 août 1665 , Lettres ..., éd. Richaudeau, II:306; 30 septembre 1665: 309-311; 29 octobre 1665: 314; lettre à Cécile de Saint-Joseph, 12 septembre 1670: 453. 
Laval s'emploiera à lui donner une très grande extension à travers le pays. ${ }^{78}$

La dévotion à l'Immaculée-Conception est définitivement implantée au pays par la consécration à la Vierge du 8 décembre 1635. Paul Le Jeune, supérieur de la mission, achève cette offrande, au nom de la Nouvelle-France, par cette prière où l'on retrouve, exprimé en des formules familières à tous ceux que nous avons cités, le but apostolique que nous avons analysé:

« Recevez, ô l'empérière [l'impératrice] des anges et des hommes, les cours de ces pauvres barbares abandonnez, que nous vous présentons par les mains de vostre glorieux Espoux, et de vos fidelles serviteurs saint Ignace et saint François-Xavier, et de tous les anges gardiens de ces misérables contrées; recevezles pour les offrir à vostre Fils, afin qu'il leur donne sa cognoissance et leur applique le mérite de son prétieux Sang. »79

Parmi les formes diverses de la dévotion à la Vierge au $17^{\mathrm{e}}$ siècle en Nouvelle-France, on doit particulièrement retenir la congrégation mariale de Québec, née en 1657,80 et la pratique dite de l'esclavage marial, déjà fort en honneur en Occident

78 Les documents sur l'activité de Mgr de Laval (1623-1708) ont été rassemblés dans les actes de son procès de béatification; sur la diffusion de la dévotion à la sainte Famille, voir notamment l'Altera nova positio super virtutibus (Rome, 1956), 220 svv. Consulter aussi l'article d'Irénée Noye sur la Sainte Famille, dans le Dictionnaire de spiritualité, V: 84-92.

79 Relation de 1635 , Divers sentimens et advis, n. 31 (éd. Thwaites, VIII : 192; éd. de Québec, I: 50). Plusieurs travaux ont été tentés sur la question: Jean-Léon Allie, o.m.i., « La Nouvelle-France et les premiers monuments de sa foi en l'Immaculée-Conception 》, dans la Revue de l'Université d'Ottawa, XII (1942), repris dans l'Oeuvre des tracts, n. 406; Adrien Pouliot, s.j., «Aux origines de notre dévotion à l'Immaculée-Conception 》, coll. Cahiers d'histoire, n. 8 (Québec, 1956). On sait que les obligations de ce vœu sont toujours observées. Marie de l'Incarnation emploie un vocabulaire tout proche de celui des Jésuites qui rédigèrent ce vœu. Dans sa première Relation, en 1633, elle parle ainsi des pécheurs: «Si j'eusse pu prendre tous ces cours et toutes ces volontés [des pécheurs], comme je le faisais d'affection, ils l'eussent bientôt aimé [Dieu]. Mais, hélas ! étant un rien comme je suis, ce que je pouvais faire était de les offrir à Dieu, afin qu'il les disposât à se donner à lui et à quitter le néant pour le Tout» (Ecrits..., éd. A. Jamet, I: 177).

80 Voir Adrien Pouliot, s.j., «Le troisième centenaire de la Congrégation de la Haute-Ville de Québec (1957) 》, dans le Rapport pour l'année 1956-1957 de La Société canadienne d'histoire de l'Eglise catholique: 103-121. 
(Espagne, France, Belgique, Pologne, et ailleurs). Plusieurs missionnaires se firent les esclaves de la Vierge; de même Catherine de Saint-Augustin, Marie de l'Incarnation, des congréganistes, etc. ${ }^{81}$ Peut-être la dévotion mariale prendra-t-elle un nouveau relief avec la fondation de la «congrégation»de Marguerite Bourgeoys, puisque ses membres doivent être «une fidèle copie de la très sainte Vierge conversant [vivant] parmi le prochain ». N'était-ce point allier dévotion et apostolat ? ${ }^{82}$

C'est cependant la dévotion au Cœur de Jésus qui semble être au Canada la plus originale. ${ }^{83}$ Les Jésuites y font allusion dans leurs lettres et dans leurs prières. Qu'il suffise d'en rappeler un seul témoin, saint Charles Garnier. Dans ses lettres à son frère carme, par exemple, la mention du Cœur de Jésus est fréquente et comme instinctive: "C'est là (en ce Cœur) où je désire habiter doresnavant $\gg,{ }^{84}$ «Je vous prie... de me placer dans le Costé de Celuy que vous tenez » (à la Messe), ${ }^{85}$ « Je vous embrasse en esprit dans le sacré Costé de Jésus-Christ ${ }^{86}$

81 A l'âge de dix ans, le 8 septembre 1642, Catherine de Saint-Augustin se consacre à la Vierge comme « esclave, servante et fille 》 (Paul Ragueneau, La vie de la Mère Catherine..., 29). Dans l'importante lettre de Marie de l'Incarnation sur ses dévotions ( vous savez bien que les dévotions extérieures me sont difficiles 》), elle confie à son fils: «Je porte au cou une petite chaîne de fer depuis plus de vingt-trois ans, pour marque de mon engagement à la sainte Mère de Dieu; je n'y ai point d'autre pratique, sinon, en la baisant, de m'offrir pour esclave à cette divine Mère 》 (lettre du 16 septembre 1661, Lettres ..., éd. Richaudeau, II: 197-200). L'esclavage marial est une pratique dévotionnelle courante au petit séminaire de Québec. Voir le détail dans le «Règlement» qui date du temps de Mgr de Laval (dans A. Pouliot, «L'extraordinaire dévotion ... .», 390).

82 La 《congrégation》que forment en 1659 les premières associées de Marguerite Bourgeoys se transforme peu à peu, avec les approbations successives de Mgr de Laval (1669 et 1676), en Institut religieux. Sur « la conformité à la vie extérieure de Notre-Dame», voir C. de Glandelet, Le vrai esprit de l'Institut des sœurs séculières de la Congrégation de NotreDame, composé en 1700-1701, et le beau chapitre $37^{\mathrm{e}}$ de dom A. Jamet dans Marguerite Bourgeoys (Montréal, 1942), 475-485.

83 Voir L. Lindsay, Les origines de la dévotion au Sacré-Cœur de Jésus au Canada (1700-1900) (Montréal, 1900).

84 Lettre du 31 mars 1636 à son frère carme (Rapport de l'archiviste... pour 1929-1930, ouvr. cité, 7; cf. F. Roustang, ouvr. cité, 288).

85 Lettre du 20 juillet 1636 à son frère carme (Rapport..., 9; F. Roustang, ouvr. cité, 291).

86 Lettre du 30 avril 1637 à son frère carme (Rapport..., 11; F. Roustang, ouvr. cité, 296). 
«Laissés-vous gouverner à ce Cour tout aimable de JésusChrist ${ }^{87}$ « Notre-Seigneur unisse votre cœur au Sien ... Regardez continuellement son Cœur $\gg, 88$ «Je prie Nostre Seigneur de tout mon cœur de posséder nos trois cours et de n'en faire qu'un avec le Sien dès à présent et dans l'éternité. ${ }^{89}$

Catherine de Saint-Augustin, dès 1663, a quelque prélibation de ce qui deviendra, douze ans plus tard, la dévotion au Cœur de Jésus à Paray-le-Monial. La dévotion de Catherine est douloureuse et tout orientée vers la réparation. ${ }^{90}$ Il en est tout autrement chez Marie de l'Incarnation. Il n'est pas indifférent de rappeler que sa dévotion au Cœur de Jésus précède notablement, comme celle de Charles Garnier d'ailleurs, la diffusion de la dévotion au $17^{\mathrm{e}}$ siècle. Les débuts de sa dévotion se situent, en effet, entre 1625 et 1635 au plus tard. Sa dévotion est fondée sur une doctrine solide, que ne voile aucun élément sentimental: elle voit et fait toutes choses, elle prie, elle adore, elle contemple, elle s'immole par et dans le Cœur de l'Homme-Dieu, tandis que sa pratique dévotionnelle consiste en la récitation d'une prière de sa façon, qui est une des effusions de cœur les plus belles, les plus profondes et les plus catholiques qui soient.91

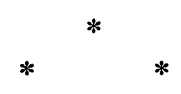

87 Lettre du 25 avril 1649 à son frère carme (Rapport..., 40).

88 Lettre du 12 août 1649 à son frère carme (Rapport..., 41; F. Roustang, ouvr. cité, 312 et 313 ).

89 Lettre commune à ses deux frères, d'après la Relation de 1650 (éd. Thwaites, XXXV: 142; éd. de Québec, II : 15) ; voir une formule à peu près équivalente dans la lettre du 25 avril 1649 .

On pourrait rapprocher de ces textes le document par lequel saint Gabriel Lalemant demandait son envoi à la Mission des Hurons, d'après la Relation de 1649 (éd. Thwaites, XXXIV: 150-154; éd. de Québec, II: 16). $90 \mathrm{Il}$ y a quelque ressemblance dans le vocabulaire des deux religieuses, bien qu'il s'agisse du Christ eucharistique chez Marguerite-Marie, et du Christ flagellé chez Catherine. «Il me dit que ce n'étoit pas moy, mais bien ses plus chers amis et les plus proches de son Coeur qui l'avoient mis en cet état 》(Paul Ragueneau, La vie de Mère Catherine..., 245).

91 Lettre à son fils, 16 septembre 1661, Lettres..., éd. Richaudeau, II : 197-202. Marie de l'Incarnation parle de la dévotion au Cœur de Jésus en bien d'autres endroits, par exemple, Ecrits ...., éd. A. Jamet, II : 314-315. On remarquera qu'elle associe, dans sa lettre de 1661, la dévotion au Cour de Jésus et celle au Cour de Marie: «Souffrez que je Vous aime par ce même Cœur...» (199). 
Ces dévotions, ${ }^{92}$ même lorsqu'elles imprègnent profondément la vie spirituelle des communautés chrétiennes, n'en constituent pourtant pas l'essentiel. La vie de la grâce c'est, pour toutes ces âmes, l'amour de Dieu qui se concrétise dans son service désintéressé. Au service de Dieu, - même lorsqu'il s'agit de «dégraisser » les jeunes Indiennes, de donner des soins aux malades atteints de la vérole ou de cabaner avec les Hurons -, le missionnaire vit en présence aussi consciente que possible de Dieu, en union avec Dieu.

Marie de l'Incarnation, reprenant sans doute une heureuse formule de saint François de Sales, parlera d'extase d'action, pour signifier que l'emploi et l'union à Dieu se réalisent en un même acte et en une même intensité de charité.93 Ses directeurs disaient qu'il fallait être «contemplatif dans l'action ». C'était bien la même chose. Tous réalisaient, à leur poste et selon leur grâce, une mystique de service. ${ }^{94}$

92 D'autres dévotions auraient pu être rappelées, plus populaires comme celle à sainte Anne et au Saint-Sacrement, plus spirituelles comme celle au Père Eternel. La dévotion au Saint-Sacrement est, par exemple, très développée chez les missionnaires, chez les Hospitalières (chez Catherine du Saint-Sacrement en particulier). Marie de l'Incarnation s'adresse habituellement au Père Eternel par le Cœur de Jésus.

93 C'est dans un très beau chapitre sur l'oraison, que les Constitutions et règlements des Ursulines de Québec déclarent: « Finalement elles sortiront de l'oraison avec une actuelle disposition de faire en perfection au moins la première chose qui se présente à faire et cela estant il y a subiect d'espérer qu'elles mériteront une grâce qui leur fera faire toutes les autres actions de la journée de mieux en mieux. Ce sont ces extases d'actions que les scurs doivent rechercher et désirer et non pas celles qui ont paru aux oraisons de quelques saintes âmes, dont elles se doivent réputer entièrement indignes 》 (fol. 342 v). Ces Constitutions, rédigées en 1647, sont l'œuvre commune de Marie de l'Incarnation et du Père Jérôme Lalemant, son directeur. Le texte manuscrit est au Vieux Monastère à Québec, il est resté malheureusement inédit.

$94 \mathrm{Je}$ reviendrai ailleurs sur la vie contemplative de Marie de l'Incarnation. Ce sujet est trop vaste et délicat pour l'aborder ici. La «pureté du cœur », le zèle apostolique, l'état de victime, l'union au Verbe incarné, les épousailles mystiques, tout l'entraîne vers un don de plus en plus désintéressé et radical aux âmes et à l'Eglise. Ce qu'elle appelle «l'esprit de JésusChrist 》 « fait courir par mer et par terre les ouvriers de l'Evangile et les fait des martyrs vivants avant que le fer et le feu les consume 》 (lettre du 22 octobre 1649, à son fils, Ecrits ..., IV: 255-256). Cette mystique de service 
Comme en d'autres temps, Dieu illustra de charismes et de grâces insignes les origines de l'Église du Canada. Les Actes des Apôtres et les Épîtres de saint Paul ${ }^{95}$ nous rapportent les événements extraordinaires qui soutinrent l'implantation de la foi dans les premières communautés et lui imposèrent comme le sceau de Dieu. Les grandes œuvres de Dieu, force nous est bien de le reconnaître, sont ordinairement frappées de ce sceau, même lorsqu'elles ont d'obscurs commencements. Il en a été ainsi en la mission de la Nouvelle-France; il en a été ainsi pour les missionnaires, pour Marie de l'Incarnation, pour Jeanne Mance, pour Catherine de Saint-Augustin, pour la bienheureuse Marguerite Bourgeoys et bien d'autres. De longue date, Dieu, par des grâces exceptionnelles, les avait préparés à leur mission.

Marie de l'Incarnation reçut, - je la cite - « de grandes lumières sur le mystère de l'Incarnation et sur l'union du Verbe avec l'Humanité sainte de Jésus-Christ », « de nouvelles lumières dans la vue des attributs divins », «la connaissance du mystère de la très sainte Trinité, dont la grandeur me fut montrée en l'unité des trois Personnes divines d'une façon tout autre que ce qui m'en avait été enseigné $\gg^{96}$ Car il y aura toujours loin de l'enseignement à l'expérience. Elle reçut encore, nous dit-elle, des «lumières très vives sur la beauté des maximes de l'Évangile », sur «le sens des psaumes », et en général sur «l'intelligence de l'Écriture Sainte ».97 Par son expérience spirituelle, qu'elle prit toujours garde de faire contrôler, Marie de l'Incarnation, véritable théologienne, a atteint une connaissance de Dieu très profonde..$^{98}$ Les charismes et grâces insignes que Dieu lui

est commune aux missionnaires de la Nouvelle-France au temps de Marie de l'Incarnation.

Consulter, sur la vie contemplative de l'Ursuline, les annotations de dom A. Jamet, l'ouvrage de P. Renaudin et celui de F. Jetté.

95 Par exemple, Actes, ch. 10, 19, etc.; Epître aux Corinthiens, ch. 12-14; Epître aux Romains, ch. 12; Epître aux Ephésiens, ch. 4. Voir l'article «Charismes », dans le Dictionnaire de spiritualité, II : col. 503-507.

96 Première Relation, dans Ecrits ..., éd. A. Jamet, I: 193, 201 et 204. 97 Ibidem, I: 166, 160; II : 308.

98 N'oublions pas qu'au dire de dom Claude Martin, Marie de l'Incarnation reçut, au titre de fondatrice, une «grâce de chef et de source »; voir La Vie de la Vénérable Mère Marie de l'Incarnation... (Paris, 1677), 402-403. Dans l'Approbation à cette Vie, Mgr de Laval affirme que « Dieu 
accordait, comme c'est le cas en de pareilles occasions, étaient donnés et reçus pour le profit spirituel de la mission.

Il y a quelques mois paraissait en France un article au titre prometteur «Le Canada français au XVII ${ }^{e}$ siècle: une société préfabriquée $\gg{ }^{99}$ La thèse est facile à établir. Les institutions françaises ont été transplantées au Canada. Le pays - la Nouvelle-France - vécut sous le régime des mêmes institutions sociales, économiques, militaires, politiques et religieuses que l'ancienne France. Le principe de cette thèse semble inattaquable. Il est non moins certain qu'il n'y a pas et qu'il ne peut y avoir de société spirituelle préfabriquée; Sigmund Diamond se garde bien de le prétendre. Il a, par ailleurs, raison d'affirmer que le cadre des institutions ecclésiastiques françaises a été transplanté au Canada.

Avec des pierres anciennes, mais qu'il a polies et ornées à sa guise, Dieu a bâti un édifice spirituel nouveau. Vivifié par un sang ancien, un corps nouveau a été animé d'un esprit nouveau. Tous ceux qui ont été employés à cette construction en ont eu plus ou moins conscience. Ils évoquent spontanément, en effet, nous l'avons vu, l'esprit de l'Église primitive. Ils se voient comme des pierres jetées dans les fondations de l'édifice que Dieu a résolu de bâtir. «O que je serois heureux d'estre un petit grain de sable jetté dans les plus creux fondemens de cette Église », déclare le Père Le Jeune, lorsque les communautés supplient qu'on les accepte au Canada. ${ }^{1}$ Et à 69 ans, Marie de l'Incarnation redira à peu près dans les mêmes termes : «Après que nous aurons fait ce que nous pourrons, nous devons nous

l'ayant choisie [Marie de l'Incarnation] pour donner commencement à l'établissement des Ursulines au Canada, lui avait donné la plénitude de l'esprit de son Institut ». Ce texte a été publié par A. Jamet, Ecrits ...., I: 117-118.

99 Sigmund Diamond, «Le Canada français au XVIIe siècle: Une société préfabriquée 》, dans les Annales, XVI (1961): 317-354.

1 Relation de 1637 (éd. Thwaites, XI: 60; éd. de Québec, 6). 
croire des servantes inutiles et de petits grains de sable au fond de l'édifice de cette nouvelle Église. $\gg^{2}$

Dieu, même lorsqu'il réemploie de vieilles pierres, fait toujours du nouveau, Ecce nova facio omnia, car il leur insuffle sa Vie. De ce souffle sont nés le sens missionnaire et ecclésial qui anima la mission de la Nouvelle-France, la joie de la croix, le désir du martyre et le martyre effectif, la ferveur dans la fidélité obscure des uns et les grâces insignes accordées aux autres pour le bien général.

C'est ainsi que Dieu fonde en renouvelant.

En ces années 1632-1672, la mission de la Nouvelle-France a vécu l'un de ses sommets spirituels, à la taille de l'avenir du pays. Paul Le Jeune, dans sa Relation de 1642, en a eu le pressentiment: «J'ai tousjours creu que le zèle d'un gouverneur [Charles Huaut de Montmagny], la bonté des François, la piété des nouveaux chrestiens, la charité des religieuses [Ursulines et Hospitalières], devoient servir de levain pour faire lever une grande masse. ${ }^{3}$

\author{
ANDRÉ RAYEZ, S.J.* \\ Séminaire «Les Fontaines》, \\ Par Chantilly (Oise), France.
}

2 Lettre à son fils, 9 août 1668, Lettres ..., éd. Richaudeau, II: 362.

3 Relation de 1642 (éd. Thwaites, XXII: 144; éd. de Québec, 24).

* Cette conférence du Père Rayez a été prononcée à la réunion générale de l'Institut, le 14 avril dernier. 